\title{
Formalizing Modularization and Data Hiding in Synthetic Biology
}

\author{
HAROLD FELLERMANN and NATALIO KRASNOGOR \\ Newcastle University \\ MAIK HADORN \\ Swiss Federal Institute of Technology \\ RUDOLF M. FÜCHSLIN \\ Zurich University of Applied Sciences
}

\begin{abstract}
Biological systems employ compartmentalization and other co-localization strategies in order to orchestrate a multitude of biochemical processes by simultaneously enabling "data hiding" and modularization. This paper presents recent research that embraces compartmentalization and co-location as an organizational programmatic principle in synthetic biological and biomimetic systems. In these systems, artificial vesicles and synthetic minimal cells are envisioned as nanoscale reactors for programmable biochemical synthesis and as chassis for molecular information processing. We present $\mathrm{P}$ systems, brane calculi, and the recently developed chemtainer calculus as formal frameworks providing data hiding and modularization and thus enabling the representation of highly complicated hierarchically organized compartmentalized reaction systems. We demonstrate how compartmentalization can greatly reduce the complexity required to implement computational functionality, and how addressable compartments permit the scaling-up of programmable chemical synthesis.
\end{abstract}

\section{INTRODUCTION}

Biological systems employ compartmentalization in order to orchestrate a multitude of biochemical processes both on an organismic level where a multitude of distinct cells communicate and interact and on a cellular level where the sub-cellular organization is prominently featured in the cytoplasm: a multitude of biochemical compounds is highly organized in vesicular compartments that co-locate reactants of desired reactions while separating those of undesired reactions. Surface markers on these compartments are used for vesicular trafficking, as well as vesicle budding and fusion [65, 66]. This allows for the fine-tuned control of cellular processes involved in e.g. biochemical synthesis, protein sorting, and endocytosis [18]. A critical review discussing the exploitation of endocytosis for the controlled uptake of nanoparticles can be found in Canton and Battaglia [12].

The desire to harvest compartmentalization as a way of providing data hiding and modular organization for next generation Synthetic Biology has led to various innovative wet lab approaches both in the area of bottom-up, as well as top-down Synthetic Biology. In the top-down approach, which generally utilizes genetically modified living systems to implement desired functionality, colonies of biological cells have been designed to perform complex molecular computations $[52,59,69,74]$. In the bottom-up approach, which employs non-living systems to achieve biomimetic behaviour, Chaplin et al. [20], for example, have demonstrated that photochromic molecules such as NitroBIPS, a kind of spiropyran, can be localized inside a collection of static polydimethylsiloxane silicone polymer (PDMS) microwells and used to construct registers, logic gates, and circuits. Other studies employed supramolecular nanoscale and microscale compartments as "nano-bioreactors" that result from the non-covalent interaction of molecules (i.e., primary self-assembly process) $[1,53-55,63]$. Several protocols have been established to control the self-assembly of these compartments into higher-order structures (i.e., secondary self-assembly process) $[3,30,32]$ as well as to induce budding [10] and fusion [17,61,75]. Even protocols for internal compartmentalization of artificial vesicles have been reported [9, 11, 36, 40, 43, 86]. More recently, Magnusson et al. [48], demonstrated the programmable assembly and disassembly of micellar nanoparticles using oligonucleotide sequences and the monitoring (e.f. using FRET) of their building and destruction in vitro and in vivo.

In the context of the synthesis of chemical products and molecular computation in programmable compartments, the ability of single vesicles to confine, transport, and manipulate hydrophilic (bio-)chemical cargo make them not only the prevalent model for natural cells [25] but also ideal as nano-bioreactors [22], drug delivery systems [77], and as starting point for the synthesis of artificial cells [58]. Assemblies of vesicles have been proposed for engineered nano-bioreactors [35], as multicomponent or multifunctional drug delivery systems [82], and as starting point for the synthesis of primitive cell communities [15]. In order to converge artificial vesicles and natural cells, single vesicles either solitary $[34,55,56]$ or incorporated in higher-order structures [30] were functionalized with artificial genetic programs and cellular components to transfer genetic blueprints into functional proteins. Oil-in-water emulsion compartments, on the other hand, offer robust, configurable, and recyclable soft colloid systems with interesting properties, and were proposed for the fabrication of delivery systems for lipophilic substances and advanced synthetic 
materials with adaptable properties [7].

The study of compartmentalization from the vantage point of information processing bears enormous potential for novel applications. If it turns out to be possible to understand the observed dynamics in compartmentalized cellular systems in terms of formal languages, the in silico development of novel therapeutic strategies can take place on high levels of abstraction and can profit from branches of mathematics and theoretical computer science that are presently not in close contact with biology or chemistry.

In this paper, we present recent research that embraces compartmentalization in synthetic biological and biomimetic systems. In Section II, we present experimental systems for programmatic compartment association and fusion. In Section III, we review state-of-the-art frameworks that are capable of expressing reactions in and of compartmentalized reaction systems, and present a novel theoretical framework that closely follows the experimental work of Section II. The novel framework is put to the test by applying it to approaches in programmable chemical synthesis as well as molecular information processing in Section IV.

\section{PROGRAMMABLE NANO-BIOREACTORS FOR SYNTHETIC BIOLOGY}

The specific and reversible in-solution self-assembly of sophisticated higher-order structures from single compartments has attracted significant attention in nano-technological applications [44, 45]. The complexity of such structures - in terms of number, type and architecture of compartments - strongly depends on the character of the linking agent. The digital nature of the DNA base coding and the specificity of binding of complementary single stranded DNA (ssDNA) oligonucleotides offer the programming of the connectivity of adjacent compartments. In the context of guiding the assembly of supramolecular compartments, the ssDNA oligonucleotides anchored to the surface of the compartments were shown to act as biomolecular combination locks, linking exclusively compartments that display complementary sequences. Using ssDNA oligonucleotides as 'smart glue' therefore allowed the programmable linkage of both hard [5, 6, 23, 51, 68, 78, 85] and soft colloids [3, 19, 29, 31, 32, 50, 71, 72]. As demonstrated in Magnusson et al. [48] even the entire compartment (in this case a small micelle) can be assembled and disassembled via DNA strand displacement.

By introducing the ligand-receptor pair biotin-streptavidin to non-covalently anchor ssDNA oligonucleotides, Hadorn and Hotz [32] presented a robust modular system to decorate the external surface of artificial vesicles as well as oil-in-water emulsion droplets Hadorn et al. [31]. By employing the specific linkage system detailed in Fig. 1 the spatially controlled DNA-directed bottom-up synthesis of complex tissue-like assemblies composed of artificial vesicles was implemented. In addition to the specified neighbor-to-neighbor interactions DNA also provided the genetic blueprint for the synthesis of functional proteins [30] (Fig. 2).

In the context of DNA-directed self-assembly, linear DNA oligonucleotides were shown to allow the self-assembly process not only to be specific but also to be reversible by a variety of external stimuli like temperature $[3,51,78]$, changes in the electrolyte concentration $[3,5]$, addition of competing oligonucleotides [76], and exploitation of the secondary structure of DNA oligonucleotides [44]. The modularity of the linking system introduced by Hadorn et al. [31] allows a secondary redecoration of the oil-in-water droplets (Fig. 3, state vi), thereby broadening the number and nature of external triggers able to reverse the DNA-guided self-assembly process of higher-order structures.

Exchange of matter and information with the surrounding medium is particularly challenging for artificial vesicles where a closed and almost impermeable membrane separates an aqueous compartment from the aqueous surrounding. [55] introduced transmembrane channels to create a selective permeability for external substances. In addition to the communication with the surrounding medium, the exchange of matter and information among compartments is another challenge when engineering cooperative chemical production in programmable bioreactors. Currently, fusion of solitary artificial vesicles that become adjacent and merge their previously individually confined content upon fusion is the main answer to this challenge $[16,26,33,73]$. In the context of this paper, the work on inducing fusion of artificial vesicles by employing ssDNA oligonucleotides anchored to the surface of the compartments is of particular interest $[19,71]$. For tissue-like assemblies of compartments, the transfer of substances is implemented for robust soft-colloid systems of printed aqueous compartments [80].

\section{FORMAL FRAMEWORKS FOR COMPARTMENTALIZED REACTIONS}

While chemistry in well-stirred reaction vessels has been described mathematically already in the late 19th century [81], formal frameworks for compartmentalized reaction systems have been suggested only within the last 15 years. The two main branches that concern themselves with compartmentalized reaction systems are $P$ systems, proposed by Paun [57], and brane calculi, proposed by Cardelli [13]. Recently, Fellermann and Cardelli [24] have developed the 


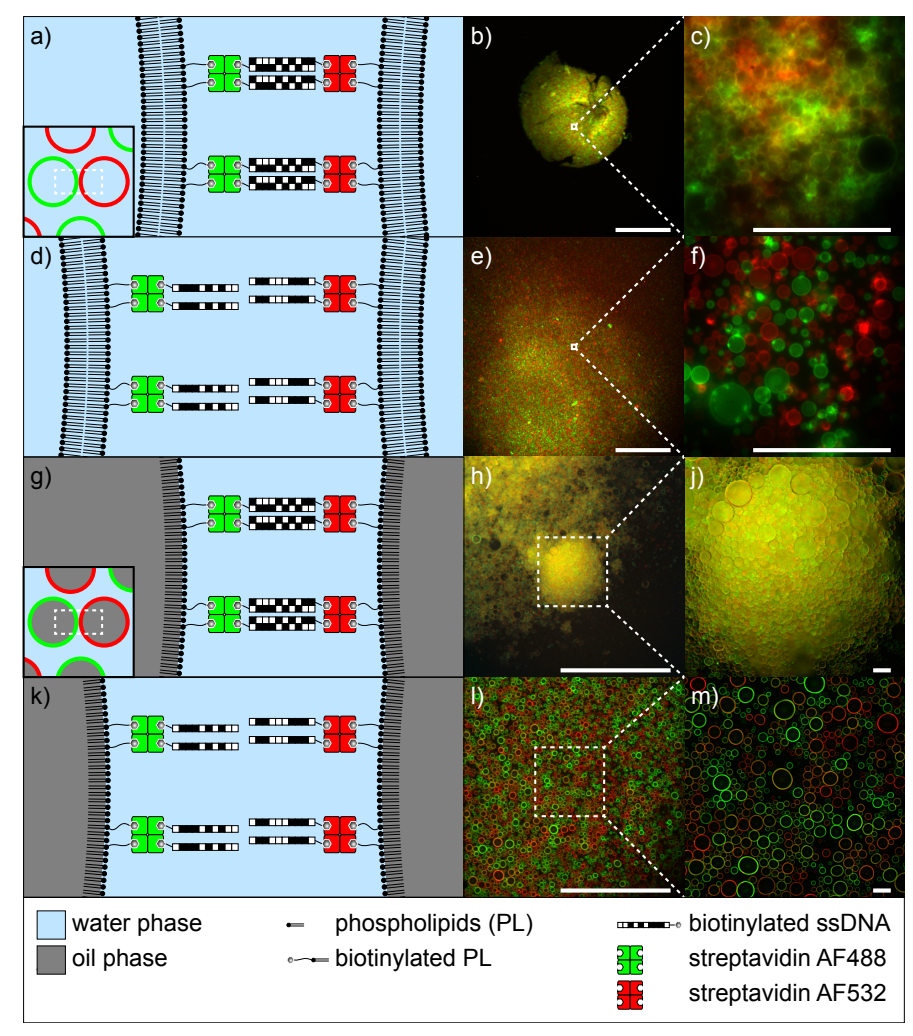

Figure 1. Specificity of DNA-mediated self-assembly of higher-order soft colloid structures. Fluorescently labeled streptavidin Alexa Fluor (AF) conjugates were used to anchor biotinylated ssDNA oligonucleotides to the biotinylated outer surface of two populations of a-f) artificial vesicles and $g-m)$ oil-in-water (o/w) emulsion droplets. The insets in a) and g) illustrate that the compartments in both cases are surrounded by an aqueous medium. Whereas the inner compartment of artificial vesicles is filled with another aqueous medium that is confined by a phospholipid bilayer (a), o/w emulsion droplets hold an oil phase that is confined by a single layer of amphiphilic phospholipids. Depending on the complementarity of the ssDNA sequences the DNA single strands combine and form a double strand. Consequently, both the vesicles and o/w emulsion droplets formed large aggregates visible to the naked eye, if the DNA sequences were complementary and link the compartments (b, h); no aggregation took place and the compartments were found solitary in case of non-complementary DNA strands (e, l). This difference in the connectivity is clearly seen in the blowups (c, f, j, m). Scale bars: $1000 \mu \mathrm{m}(\mathrm{b}, \mathrm{e}, \mathrm{h}, \mathrm{l}), 50 \mu \mathrm{m}(\mathrm{c}, \mathrm{f}, \mathrm{j}, \mathrm{m})$. Fluorescence micrographs b, c, e, f modified from Hadorn et al. [30]; h, j, l, m modified from Hadorn et al. [31].

chemtainer calculus in an attempt to bring the theoretical framework of brane calculi closer to a potential experimental implementation in a biomimetic setup.

\section{A. P Systems and Brane Calculi}

All mentioned frameworks employ a language of balanced parentheses to capture the topological organization of nested compartments. Each compartment can hold a multiset of arbitrary chemicals as well as other compartments. In $P$ systems, compartments are traditionally labeled and the molecular content is given by associating a multiset of molecules to each compartment label. In brane calculi, the grammar for parentheses is extended to include molecules directly. Thus, the formal language is defined by the recursive production rules

$$
\mathrm{P}:=\emptyset: \mathrm{P}+\mathrm{P}: \& \mathrm{P} D: m_{j}
$$

for the non-terminal and start symbol $\mathrm{P}$, the terminals $\{\emptyset,+, \diamond, D\} \cup\left\{m_{j} \mid j \in J\right\}$ and the broken vertical bar indicating choice. Here, $\mathcal{M}=\left\{m_{j} \mid j \in J\right\}$ is a set of chemicals for some index set $J$ and the halfmoon parentheses denote compartments. Structural equivalence relations are introduced to give $(\mathcal{M},+, \emptyset)$ associative and commutative semantics with the neutral element $\emptyset$.

Over the state space defined by the grammar in Eq. (1), chemical reactions can be introduced as bulk reactions

$$
P \longrightarrow P^{\prime}
$$




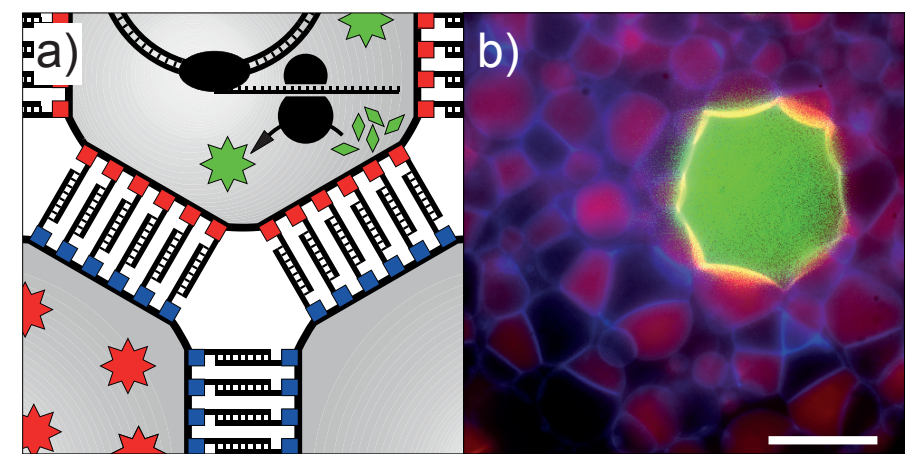

Figure 2. Protein synthesis in macroscopic assemblies of microscopic nano-bioreactors. a) DNA strands provide the genetic blueprint for the synthesis of a fluorescently active protein (green star) as well as define the connectivity of three distinct populations of man-made microscopic compartments. b) The protein synthesizing containers (green) are integral part of macroscopic higher-order assemblies of containers (red, black) that result from a self-assembly process. Scale bar: $20 \mu \mathrm{m}$. Figure modified from Hadorn et al. [30].

for multisets $P=\sum_{i} \nu_{i} m_{i}$ and $P^{\prime}=\sum_{j} \mu_{j} m_{j}$ of simple educt and product molecules with stoichiometric coefficients $\nu_{i}$ and $\mu_{j}$. In addition, reactions can describe trans-membrane processes

$$
P+\varangle Q D \longrightarrow P^{\prime}+\varangle Q^{\prime} D
$$

where $P, P^{\prime}, Q, Q^{\prime}$ are again simple multisets of molecules. For example, the action of a sodium-potassium pump could be codified by the reaction

$$
2 \mathrm{~K}^{+}+83 \mathrm{Na}^{+}+\mathrm{ATP} D \longrightarrow 3 \mathrm{Na}^{+}+\varangle 2 \mathrm{~K}^{+}+\mathrm{ADP} D .
$$

In traditional P systems, all compartments of a membrane structure are labeled and each compartment has its specific set of reaction rules. Brane calculi also associate reaction rules with individual membranes but employ a dedicated syntax that directly attaches the rule to the respective membrane. For example, reaction (3) would be associated to some compartment with content $R$ by writing

$$
! P(Q) \triangleright P^{\prime}\left(Q^{\prime}\right) \& R D .
$$

The reaction can occur if and only if $P \subset R$. Importantly, the brane calculus defines a process algebra for sequential and parallel composition of rules. For example, the exclamation mark in the above notation signifies that the rule is not consumed upon application.

Avoiding explicit labeling of compartments has the advantage that one can introduce reactions which fundamentally alter the arrangement of the compartments themselves, such as compartment fusion

$$
\text { mate }_{i}\left(P D+\operatorname{mate}_{i}^{\top}(Q D \longrightarrow ß P+Q D\right.
$$

where two compartments with contents $P$ and $Q$ fuse to form a single compartment of mixed content. Fusion is triggered by a mate action associated with one compartment and a respective co-action associated with the other compartment. Compartments fuse if and only if the two actions match. Similarly, a drip action can initiate compartment fission:

$$
\operatorname{drip}_{B} P+Q D \longrightarrow B P D+\& Q D
$$

Note that this brief summary barely touches the surfaces of both frameworks. For a complete introduction to $\mathrm{P}$ systems and brane calculi the reader is advised to consult Paun [57] and Cardelli [13]. Although both frameworks where originally developed with non-deterministic semantics, Bacci and Miculan [2] have recently developed stochastic semantics that allows for their use e.g. in stochastic simulation.

\section{B. The Chemtainer Calculus}

Closely following the experimental accomplishments of Stengel et al. [71], Chan et al. [19], Hadorn and Hotz [32], and Hadorn et al. [31], the chemtainer calculus employs membrane associated DNA markers to govern the fate of 


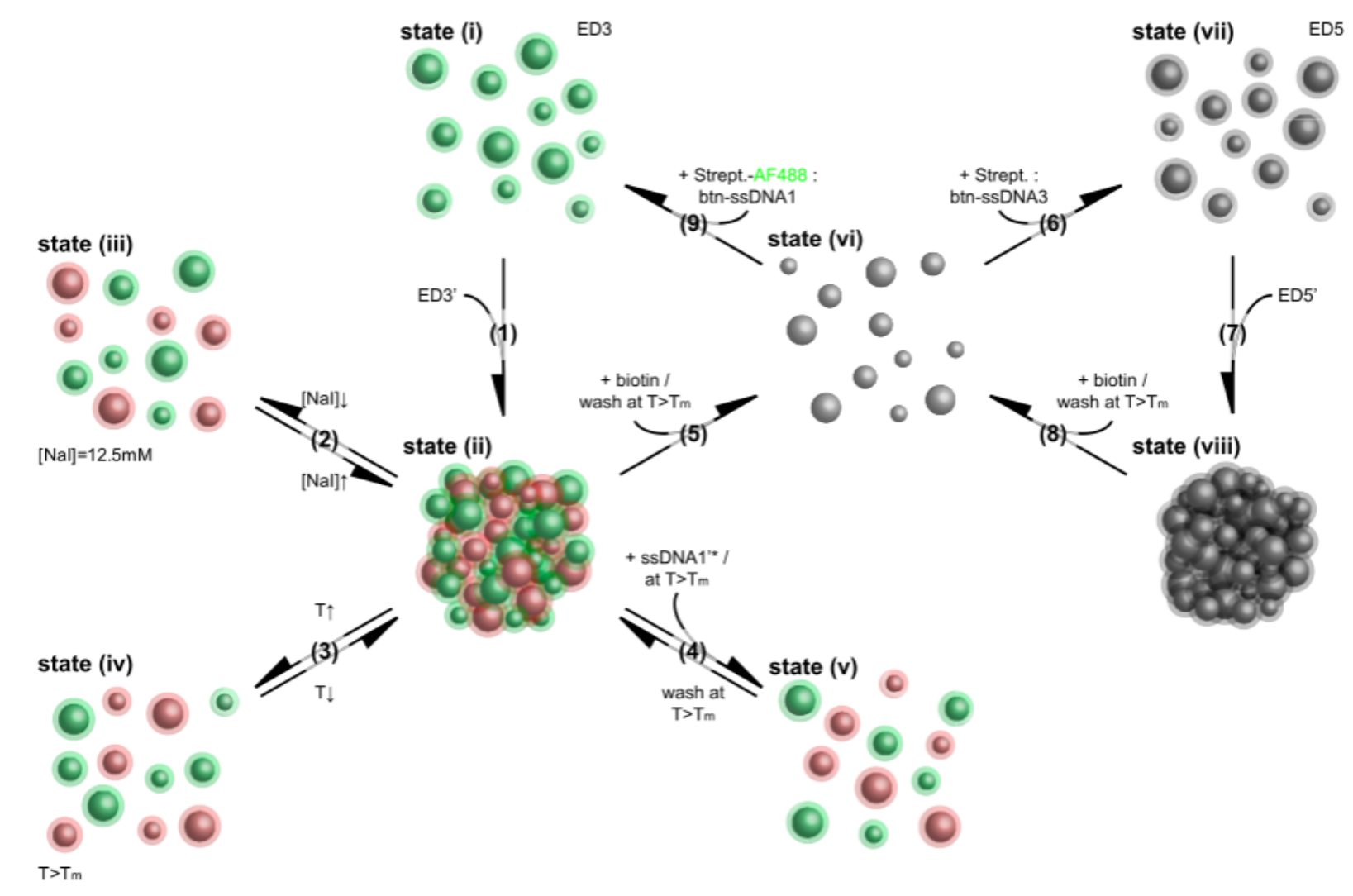

Figure 3. Specificity and reversibility of the self-assembly process and recyclability of oil-in-water (o/w) emulsion droplets. The DNA-mediated self-assembly of o/w emulsion droplets was reversed by decreasing the salt concentration of the external aqueous medium (2) and by increasing the temperature (3); both caused the DNA double strands to break apart. By introducing a short DNA single strand not immobilized on the surface of the o/w emulsion droplets, one of the binding DNA oligonucleotides was replaced and the assemblies broke apart (4). Stripping off the streptavidin molecules that anchored the DNA oligonucleotides to the $\mathrm{o} / \mathrm{w}$ emulsion droplet surface not only caused the assemblies to disintegrate (state vi) but allowed the redecoration of the $\mathrm{o} / \mathrm{w}$ emulsion droplets using a different combination of streptavidin and single stranded DNA (state vii). These newly decorated o/w emulsion droplets were subsequently able to undergo aggregation with another droplet populations previously not available for aggregation (state viii). Figure taken from Hadorn et al. [31].

supramolecular compartments. The chemtainer calculus uses two different types of DNA markers: on the one hand, simple ssDNA tags are used for compartment recognition and fusion similar to the mate action in the original brane calculus:

$$
{ }^{\sigma} B P D+{ }^{\sigma^{\top}} B Q D \longrightarrow{ }^{\sigma \|} B P+Q D
$$

Here, $\sigma$ signifies a DNA single strand with a specific sequence, $\sigma^{\top}$ its complement, and $\sigma^{\|}$the conjugated double strand. Hybridization of the double strand is controlled by temperature, and the two single strands are recovered when surpassing the melting temperature of the double strand:

$$
\sigma^{\sigma^{\|}} \& P D \longleftrightarrow{ }^{\sigma+\sigma^{\top}} \text { \& } P D
$$

Join gates, on the other hand, are multi-strand DNA constructs that allow for the specification of DNA-based computational processes $[14,21,42]$. In this approach to DNA computation, one DNA strand that is composed of several logical domains, with all but one domain being hybridized to one or more complementary strands. The only initially exposed single strand domain of the gate is a short toehold region. This toehold can reversibly bind a complementary signal strand which is designed to be longer than the toehold domain and complementary to the next domain(s) of the template. The newly binding signal is then able to hybridize to all matching domains of the template, thereby displacing strands that where previously bound and possibly exposing new toeholds. The displaced strands can either be output signals, or signals that bind to toehold regions of downstream gates. By choosing domains of appropriate length, it can be guaranteed that toehold binding is reversible, whereas the total strand displacement 
process is effectively irreversible, thus computation is energetically downhill and kinetically irreversible, if and only if all correct input strands are present.

Disregarding the details of strand displacement, the chemtainer calculus encodes join gates with the syntax

$$
s^{*} \triangleright s^{\prime}
$$

where $s^{*}$ denotes the set of input strands that the gate recognizes, and $s^{\prime}$ denotes the gates' output strand. The action of the gate is then given by the transition

$$
s^{*} \triangleright s^{\prime}+s^{*} \longrightarrow s^{\prime}
$$

That is, the gate exposes its output strand $s^{\prime}$ if and only if all its inputs are present, and inputs are consumed upon firing of the gate. Just like simple DNA tags, join gates are membrane bound.

Note that the original chemtainer calculus uses slightly different fusion semantics, has the additional notion of physical locations, and defines a small programming language for external nano-bioreactor manipulation. Here, we restrict ourselves to the elements of the calculus used in the applications that follow. For a concise exposition, the reader is referred to Fellermann and Cardelli [24].

\section{APPLICATIONS}

We now demonstrate how the chemtainer calculus, brane calculus, and $\mathrm{P}$ systems can be used to design and analyze compartmentalized reaction systems in bottom-up, middle-out, and top-down Synthetic Biology. The examples address chemical synthesis (Section IVA) and molecular computing (Sections IVB and IV C) in vesicles and synthetic cells. We also contrast the fusion-based approach to an alternative approach that relies solely on compartment communication (Section IV C) and association (Section IV D).

\section{A. Programmable Chemical Synthesis}

Fellermann and Cardelli [24] and Weyland et al. [83] have employed the chemtainer calculus for the programmed synthesis of oligosaccharides - a computational problem first introduced by Reller [60]. Oligosaccharides are branched heteropolymers composed of typically five to ten individual sugar monomers such as mannose, galactose, and glucose. This diverse class of biochemicals is involved in various physiological processes pertaining e.g to cell-cell recognition, intra- and intercellular trafficking, and metabolic modulation [79].

The synthesis of such molecules is difficult, because the non-linear structure of the target molecules does not allow a basically linear progression as in the case of protein synthesis (we neglect post-processing, the statement refers only to the translation of mRNA into chains of amino acids). Such linear synthesis of a chain of monomers by adding one monomer after the other is - from a pure control perspective - quite simple because it allows for the use of templates. For branched polymers, the usage of templates would be much more complicated to implement, because either one would have to deal with a multitude of "construction sites", or an agent, such as the ribosome, would have to jump between these sites.

If one cannot rely on templates, the structure of the target molecules has to be encoded in the linkers between the monomers that constitute the building blocks of the target. Synthesis via self-assembly would be easy if the linkers between the monomers are highly selective such that essentially each link carries its own specific address (for an implementation of such a self-assembly process on a DNA based supra-molecular scale, see Rothemund [64]). However, for most branched polymers, the linkers have to be small in spatial extension which in practice limits selectivity. "Click chemistries" introduced by Best [4], Kolb et al. [38], Kolb and Sharpless [39], Kurpiers and Mootz [41] offer a way to implement the self-assembly of branched polymers using a small set of highly reliable and selective reactions for the rapid synthesis of new compounds through heteroatom links. The concept of click chemistries allows not only the addition of monomers to an already existing polymer, but the linkage of partially assembled polymers. In any case, the problem of side reactions in the synthesis of branched polymers, as described in Koeller and Wong [37] is still present, if the self-assembly process takes place in a homogeneous reaction environment.

The number of potential side products can be minimized, however, by controlling the exact order by which reactants and intermediate products of a complex reaction cascade are co-located. For example, assume that the structure $P_{3}$ 


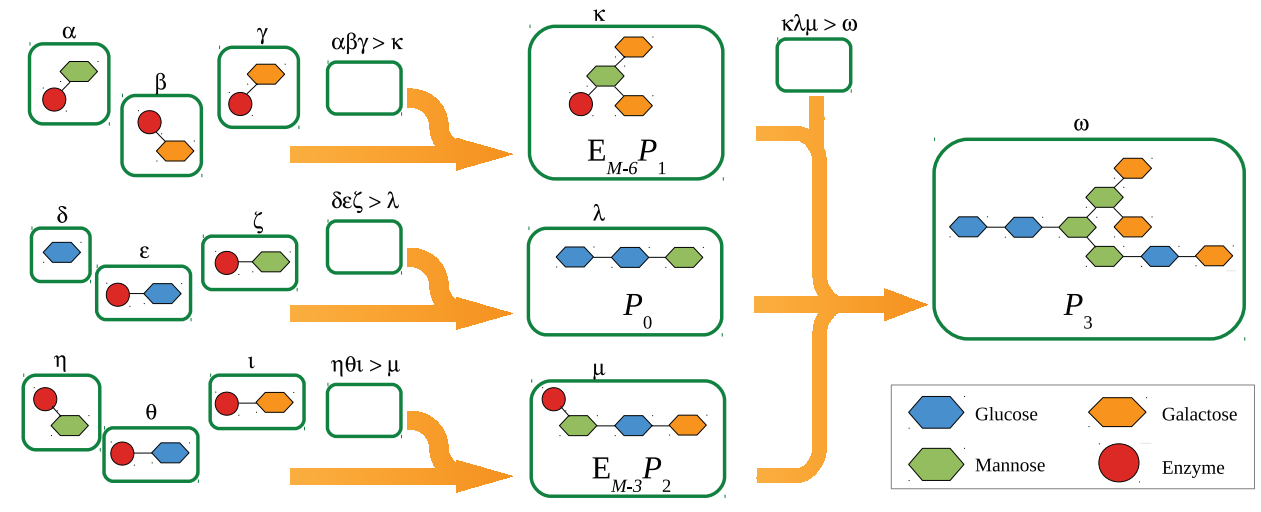

Figure 4. Programmed chemical synthesis of the target oligosaccharide $P_{3}$ by subsequent co-location of intermediate reactants. Green outlines indicate compartments and orange arrows show chemical reactions triggered by programmed compartment fusion. Figure modified from Fellermann and Cardelli [24].

shown in Fig. 4 can be produced with the reaction cascade

$$
\begin{aligned}
& \mathrm{Gal}+\mathrm{E}_{\mathrm{Gal}-4}^{*} \mathrm{Gal}+\mathrm{E}_{\mathrm{Gal}-4}^{*} \mathrm{Man} \longrightarrow P_{0}+2 \mathrm{E}_{\mathrm{Gal}-4} \\
& \mathrm{E}_{\text {Man-6 }}^{*} \mathrm{Man}+\mathrm{E}_{\text {Man-6 }}^{*} \mathrm{Glc}+\mathrm{E}_{\text {Man-3 }}^{*} \mathrm{Glc} \longrightarrow \mathrm{E}_{\text {Man-6 }}^{*} P_{1}+\mathrm{E}_{\mathrm{Man}-6}+\mathrm{E}_{\mathrm{Man}-3} \\
& \mathrm{E}_{\mathrm{Man}-3}^{*} \mathrm{Man}+\mathrm{E}_{\mathrm{Man}-2}^{*} \mathrm{Gal}+\mathrm{E}_{\mathrm{Gal}-4}^{*} \mathrm{Glc} \longrightarrow \mathrm{E}_{\mathrm{Man}-3}^{*} P_{2}+\mathrm{E}_{\mathrm{Man}-2}+\mathrm{E}_{\mathrm{Gal}-4} \\
& P_{0}+\mathrm{E}_{\mathrm{Man}-6}^{*} P_{1}+\mathrm{E}_{\mathrm{Man}-3}^{*} P_{2} \longrightarrow P_{3}+\mathrm{E}_{\mathrm{Man}-6}+\mathrm{E}_{\mathrm{Man}-3},
\end{aligned}
$$

where each reaction combines three reagents to create either an intermediate or the final product. It has to be ensured that reactions (12) through (14) occur in isolation and prior to reaction (15) in order to avoid undesired side products. Weyland et al. [83] present an algorithm that identifies such optimal reaction cascades.

Assuming that all initial reactants are provided in uniquely DNA tagged reaction compartments, we can introduce a single empty compartment which is decorated with complementary DNA strands as well as join gates. While the simple DNA tags orchestrate co-location of reactants via fusion, join gates reflect the outcome of the respective chemical reaction by altering the DNA markers on the surface. This is shown here for reaction (12):

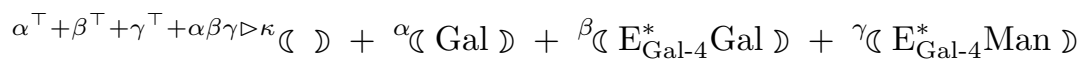

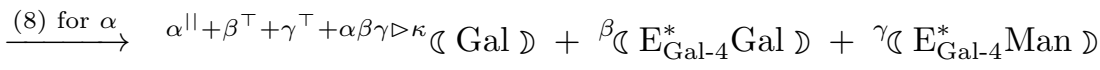

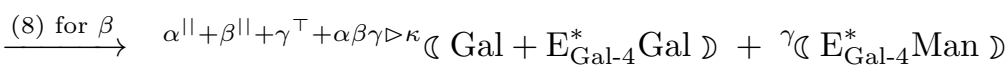

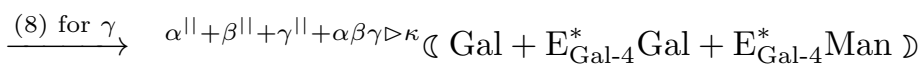

$$
\begin{aligned}
& \stackrel{3 \times(9)}{\longrightarrow} \alpha+\alpha^{\top}+\beta+\beta^{\top}+\gamma+\gamma^{\top}+\alpha \beta \gamma \triangleright \kappa / \mathrm{Gal}+\mathrm{E}_{\mathrm{Gal}-4}^{*} \mathrm{Gal}+\mathrm{E}_{\mathrm{Gal}-4}^{*} \mathrm{Man} D \\
& \stackrel{(11),(12)}{\longrightarrow} \alpha^{\top}+\beta^{\top}+\gamma^{\top}+\kappa\left(P_{0}+2 \mathrm{E}_{\mathrm{Gal}-4}\right) \text {. }
\end{aligned}
$$

The newly exposed $\kappa$ tag signifies that the compartment is ready for downstream processing. Here, the complementary tags $\alpha^{\top}$ a.s.o. are effectively garbage, and could easily be avoided by feeding the join gate $\alpha \alpha^{\top} \beta \beta^{\top} \gamma \gamma^{\top} \triangleright \kappa$ instead.

Noteworthy, from a standard library of monomers, we can control a reaction cascade to obtain any desired target compound simply by providing compartments that are decorated with appropriate DNA tags and gates. Paired with automatized equipment such as liquid handling robots and microfluidic technology, this opens up for truly programmable chemical synthesis.

\section{B. Modular Molecular Computing}

Romero-Campero et al. [62] and Smaldon et al. [70] construct molecular logical gates from gene regulatory networks. Each gene features a promoter site, an operator site, and a protein encoding region, with proteins that serve as repressors by binding to operator sites of other genes. The authors demonstrate that such operons can serve as NOT 

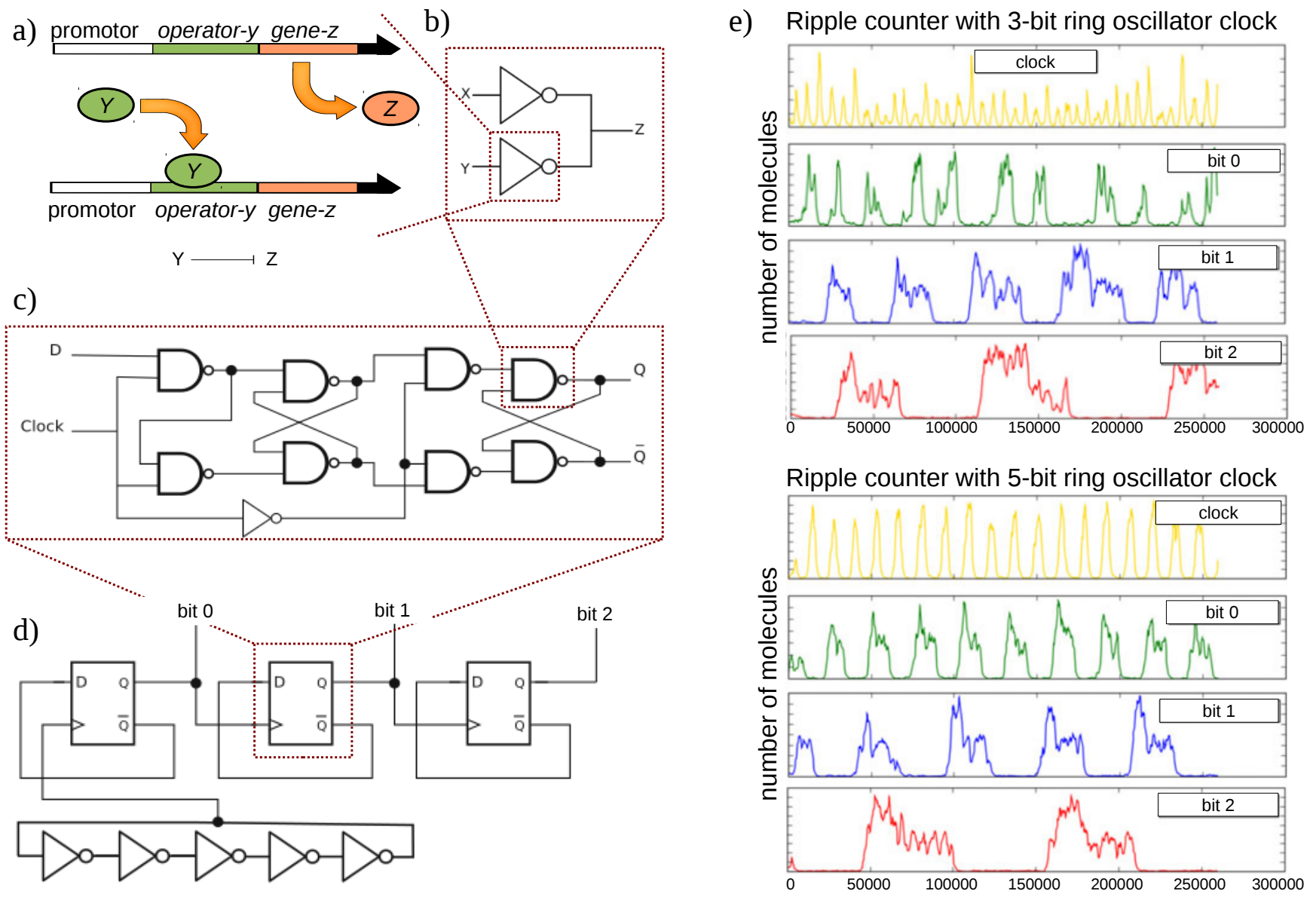

Figure 5. Gene regulatory network implementation of a 3-bit ripple counter by Smaldon et al. a) Modular circuits are built from operons comprising a promoter, an operator site, and a gene encoding region. In the absence of the repressor protein $Y$, protein $Z$ is expressed. In the presence of $Y$, protein $Z$ is repressed. Thus, the operation implements a NOT gate. b) Two NOT gates in parallel build a NAND gate. c) Eight NAND gates and one NOT gate can be wired to form a D-flipflop. d) Three D-flipflops and a 5-bit ring oscillator built from serial NOT gates form the 3-bit ripple counter. e) Trajectories of the output bits obtained from stochastic simulation show that the ripple counter operates reliably with a 5-bit ring oscillator clock (lower data set) whereas the flipflops sometimes fail to switch when driven by a faster, 3-bit ring oscillator clock (upper data set). Figure modified from Smaldon et al. [70].

gates and proceed to construct modular gates of successively higher complexity (c.f. Fig. 5). The most complex example is a 3-bit ripple counter implemented by 56 operons that express 32 mRNAs and corresponding proteins. The overall circuit is thus composed of 120 molecular species. It is specified using $\mathrm{P}$ systems and compiled into, and simulated using, a Dissipative Particle Dynamics engine.

Although the authors study the effect of encapsulating logical gates into single vesicles, the full advantage of compartmentalization becomes apparent when distributing individual logical gates over several compartments. Inspecting the structure of the ripple counter in Fig. 5.d shows that the circuit is built from three D-flipflops and one 5-bit ring oscillator - each of them being built from more elementary modules. While the D-flipflop circuit, for example, is built from 17 operons that express nine distinct proteins, it is wired to other modules only by two repressor proteins - one serving as input and the other one as output. The remaining seven regulator proteins serve only for internal wiring of the module.

This observation motivates a circuit design where modules are encapsulated into separate compartments which are permeable for molecular species that wire modules among each other, while being impermeable for species that wire gates within each module. Doing so allows to reuse the same molecular species for multiple instances of a module type, e.g. the D-flipflops in the ripple counter. This can drastically reduce the number of required molecular species.

For the 3-bit ripple counter, we choose permeable species for the clock output signal and the three counter bits. A 
a)

b)

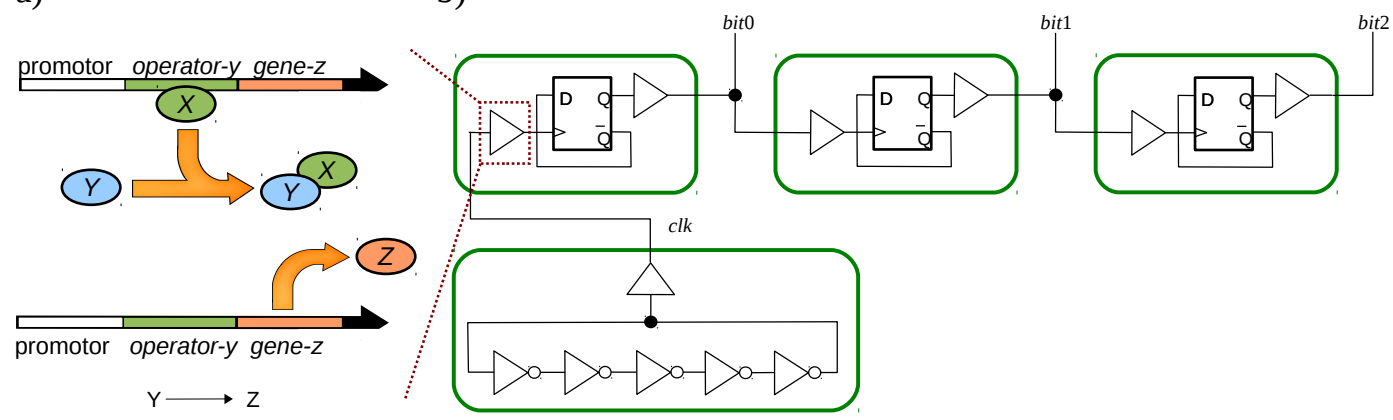

Figure 6. a) Transducers are built from un-inhibiting operons: in the absence of the activator protein $Y$, an inhibitor $X$ suppresses gene expression. In the presence of $Y$, the inhibitor $X$ dissociates from the operon and protein $Z$ is expressed. b) Transducers are used in the compartmentalized reimplementation of the ripple counter from Fig. 5.d. Green lines indicate compartment boundaries, and only molecules representing clk, bit0, bit1, and bit2 are permeable.

naïve reimplementation of the circuit shown in Fig. 5.d would still require us to use distinct DNA sequences for five of the 17 operons that make up each flipflop, because the module input and output is wired to five operons per flipflop. We can do better by adding transducer gates at the input and output of the original flipflops. One transducer senses an input signal that corresponds to a permeable species, and expresses the flipflop's original input, which is now also an impermeable species. The second transducer senses the original output signal and expresses a permeable output signal. Although the number of operons per gate now increases from 17 to 19, the total number of operons decreases from 56 to 28 because operons forming a flipflop module can be reused without modification. The resulting circuit and its encapsulation is shown in Fig. 6.

We now employ the chemtainer calculus to assemble the different modules of the ripple counter and their wiring from a library of general parts. Our strategy is to provide a set of compartments that contain flipflops, and counters without any external wiring, and fuse them with compartments that contain transducers to implement the specific wiring among modules.

For the case of a flipflop $F(x, y)$ with original (impermeable) input $x$ and output $y$, and two transducers $T(c l k, x)$ and $T(y, b i t 0)$ for the permeable species $c l k$ and bit0, the corresponding operation in the chemtainer calculus reads:

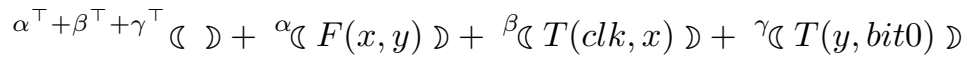

$$
\longrightarrow \alpha^{\alpha^{\|}+\beta^{\|}+\gamma^{\|}} \text {} S F(c l k, \text { bit0 }) D \text {. }
$$

Following this procedure, we can generate the entire ripple counter

$$
\varangle C(c l k) D+\varangle F(c l k, b i t 0) D+\varangle F(b i t 0, b i t 1) D+\varangle F(b i t 1, b i t 2) D
$$

where we have omitted the DNA tags for simplicity.

Furthermore, compartments with intricate internal logic, such as the flipflop ${ }^{\alpha} \zeta F(x, y) D$ or clock ${ }^{\delta} \zeta C(x) D$ can be provided as a general library, whereas compartments that determine the specific wiring, such as ${ }^{\beta}(C(c l k, x) D$ comprise only one internal and one surface associated DNA strand.

\section{Modular Bacterial Computing}

The utilization of robust ways for encoding and enforcing compartmentalization is not restricted to bottom-up or middle-out Synthetic Biology. Indeed, in the top-down approach to Synthetic Biology, there is an equal drive to pursue "divide and conquer" strategies as a way of composing computational circuits from a small library of parts. For example, Tamsir et al. [74] and Regot et al. [59] have used as "compartments" entire cells with engineered gene regulatory networks that implement elemental logical operations. As in the last section, increasingly complex circuits are built from this set of elemental gates by co-locating and wiring cells with the required functionality. In order to implement this wiring, Tamsir et al. [74] exploit the quorum sensing mechanism of Escherichia coli to signal logical information from one cell to another. Notably, all colonies of E. coli carry the same NOR gate, but the inputs and outputs are wired to different orthogonal quorum-sensing molecules. The authors demonstrate that all 16 two input 
a)

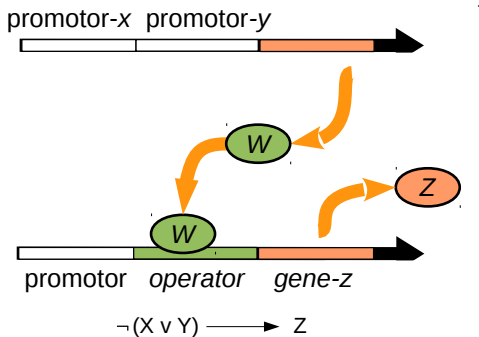

b)

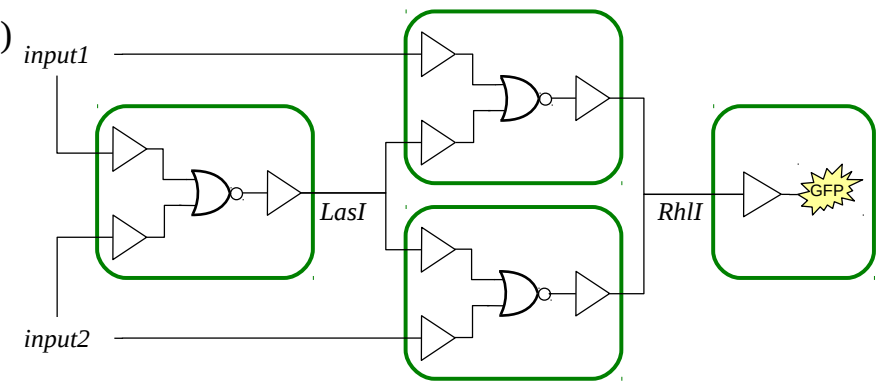

Figure 7. Implementation of a distributed XOR gate in colonies of E. coli by Tamsir et al. [74]. a) NOR gates are implemented by two operons: the first operon is regulated by a tandem promoter for the two input signals and codes for a repressor of the second operon. Thus, gene $z$ is only expressed from the second operon if both inputs $x$ and $y$ are absent. b) The distributed implementation of the XOR gate from three NOR gates and one reporter cell. The NOR gates are incorporated into bacterial colonies and wired to different quorum sensing devices (which perform as transmembrane signal transducers) that perform communication among modules. The final output of the XOR gate triggers expression of GFP in the reporter cell. Figure modified from Tamsir et al. [74].

Boolean functions can be implemented by no more than four different E. coli cells (see Fig. 7 for an implementation of the XOR gate).

Communication among cells can be easily formalized using P systems or the brane calculus. The original implementation of the chemtainer calculus does not feature transmembrane reactions as in equation (3). We give a formalization of the XOR gate in the brane calculus. Each NOR gate can be encoded by the following transitions:

$$
\begin{array}{cl}
\text { promoter binding: } & t_{1}:=(x+a) \longleftrightarrow(x: a) \\
& t_{2}:=(y+a) \longleftrightarrow(y: a) \\
\text { gene regulation: } & t_{3}:=(b+w) \longleftrightarrow(b: w) \\
\text { gene expression: } & t_{4}:=(x: a) \longrightarrow(x: a+w) \\
& t_{5}:=(y: a) \longrightarrow(y: a+w) \\
& t_{6}:=(b) \longrightarrow(b+z)
\end{array}
$$

In the above, $a$ and $b$ denote the two genes, $x$ and $y$ the inputs, $z$ the output, and $w$ the repressor protein. Colons denotes molecular association and the parentheses indicate that the transitions occur inside compartments. The entire NOR gate is given as the parallel composition of the above transitions, namely:

$$
\mathrm{NOR}:=t_{1}\left|t_{2}\right| t_{3}\left|t_{4}\right| t_{5} \mid t_{6}
$$

with the vertical bar denoting parallel composition (see Cardelli [13] for a formal definition).

The NOR gate is now reused in the different synthetic E. coli strains and wired to the respective quorum sensing devices. In brane calculi, the resulting cell definitions read (compare with Fig. 7):

$$
\begin{aligned}
& \text { Cell1 }:=\operatorname{lin} 1() \triangleright(x)|! \operatorname{in} 2() \triangleright(y)| !(z) \triangleright \operatorname{LasI} \mid ! \mathrm{NOR}_{(} a+b \triangleright \\
& \text { Cell2 }:=\operatorname{lin} 1() \triangleright(x)|! \operatorname{LasI}() \triangleright(y)| !(z) \triangleright \operatorname{RhII} \mid ! \mathrm{NOR}(a+b) \\
& \text { Cell3 }:=\text { !LasI }() \triangleright(x)|! \ln 2() \triangleright(y)| !(z) \triangleright \operatorname{RhII} \mid ! \mathrm{NOR}_{(} a+b \text { D } \\
& \text { Cell1 }:=! \operatorname{RhlI}() \triangleright(\mathrm{GFP}) \& D
\end{aligned}
$$

The distributed XOR gate results from the combination of all bacteria:

$$
\text { XOR }:=\text { Cell1 }+ \text { Cell }+ \text { Cell } 3+\text { Cell } 4
$$

Based on this formalization, stochastic simulations of the bacterial computational process are straight forward [2].

Signaling via quorum sensing molecules as well as via pheromones (as exemplified by Regot et al. [59]) has the disadvantage that the limited number of wiring molecules does not easily open up for scalable circuits. Regot et al. [59] analyzed different implementations of all three input Boolean functions and identified that a library of elemental AND, N-IMPLIES, IDENTITY, and NOT gates would produce the best building blocks for implementing more complex circuits with the minimal number of chemical wiring species. A promising alternative has been introduced by 

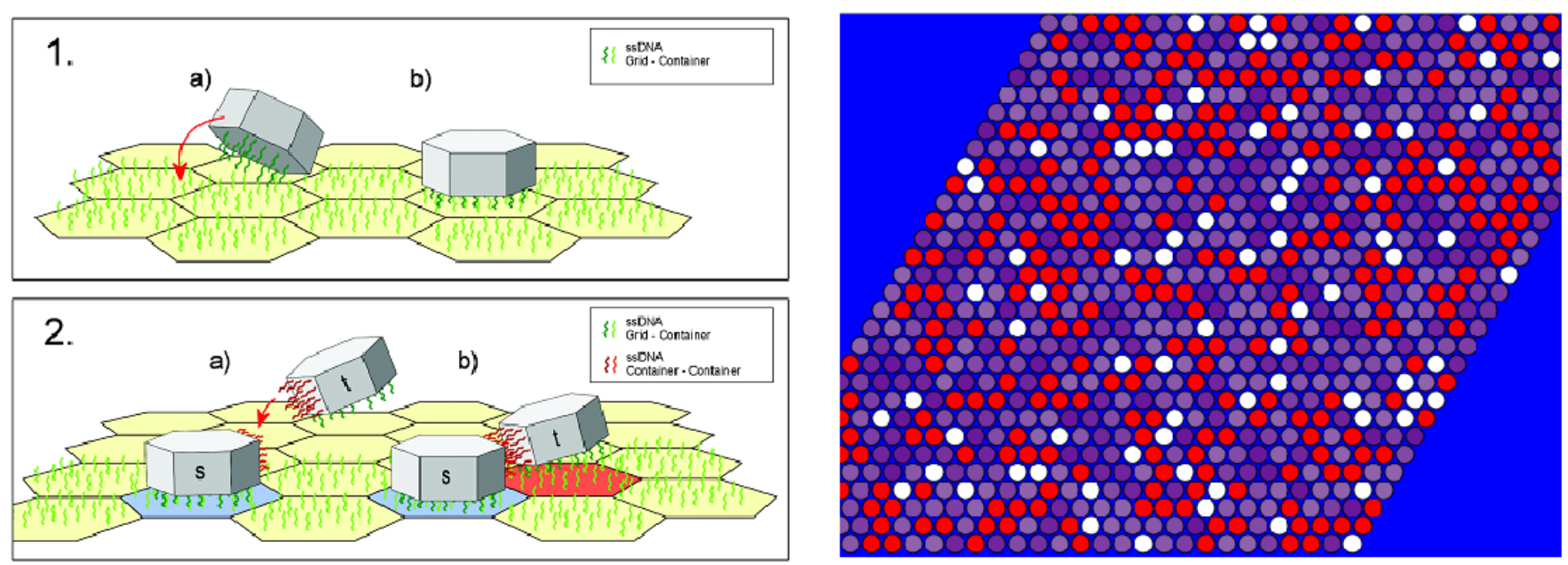

Figure 8. Self-assembly of a $2 \mathrm{D}$ grid of compartments via specific ssDNA linkers (left). Compartments assemble by weak nonspecific association to the grid (1) and stronger specific association to complementarily tagged compartments (2) An example of a spatially heterogeneous microreactor is shown on the right. Each circle represents a single compartment and the colors indicate different container types. Figure taken from Reller [60].

Silva-Rocha and de Lorenzo [69], who demonstrate how aromatic metabolites of, in their case, Pseudomonas putida cells can be used as molecular wires. As catabolic pathways of aromates are specific to certain kinds of organisms, this approach holds the promise that metabolic wires are orthogonal to the metabolism of host species, so that no crosstalk would be expected with the endogenous metabolic networks of distant hosts.

\section{Programmable Nano-Bioreactor Tissues}

In sections IV A and IV B, compartment markers have been exclusively used to initiate fusion. As an alternative approach, Reller [60] considers a system where DNA markers dictate not the fusion but the spatial self-assembly of compartments into heterogeneously arranged reaction environments which can be regarded as an artificial tissue system. In the cited in silico investigation, such a tissue was used for the synthesis of branched polymers, e.g. smaller oligosaccharides.

The purpose of an artificial tissue system is to reduce the number of potential side reactions by (at least partially) controlling the temporal sequence of reaction steps by spatial organization of the reaction environment. In Reller [60] and later in Fuchslin et al. [27], an approach was presented in which compartments assemble into a reaction environment, an artificial tissue. This tissue then hosts a second assembly step, namely the assembly of branched polymers.

In the assembly step building up the tissue, compartments are assumed to either freely float above the substrate (here a hexagonal grid) or bind to the ground as well as to already bound adjacent compartments via DNA surface markers (c.f. Fig. 8). Importantly, bonds between compartments are reversible, such that a bound compartment can be released again. The importance of reversibility is discussed by Whitesides and Boncheva [84]. We assume that binding to the substrate is non-selective and relatively weak. Where available, binding to neighbors is reversible and selective with regard to the DNA markers. If a compartment is connected to six matching neighbors, the total binding energy is tuned such that the release is no longer likely. This (reversible) self-assembly process is run long enough to ensure the emergence of a structure with a defined neighborhood correlation, though not with a spatially regular pattern (Fig. 8, right).

The resulting spatially organized tissue can be used for chemical synthesis as well as for molecular computing. For both scenarios, it is assumed that different types of compartments (identified by their DNA markers) provide distinct chemical environments, for example for catalyzing the formation or breaking of some of the possible links between the molecular monomers depicted in Fig. 4. One way of realizing this is by anchoring catalysts to the inner compartment membranes. Whereas the chemical environment of a compartment is fixed, other molecular content is allowed to diffuse among adjacent compartments, for example by means of membrane pores or gap junctions.

Reller [60] applies this approach to chemical synthesis of branched heteropolymers, as discussed in Section IV A. The authors perform stochastic simulations of both the tissue self-assembly and chemical synthesis processes. For each 
resulting tissue system, the measured yield of a given target compound is used as fitness function of an evolutionary algorithm that optimizes the composition of compartment types, i.e. the chemical functionality and surface decoration of compartments. The study demonstrates in silico that it is possible to create a spatially heterogeneous reaction environment with a bias towards specific reaction channels that increase the yield of the target compound over the one obtained in a one-pot reaction up to three orders of magnitude for specific cases of tissues with almost perfect spatial structuring and one order of magnitude for tissues with neighborhood correlation between different types of compartments but no obvious direct patterning. Importantly, this increase is purely due to spatial organization and happens without any alteration of the kinetic constants for the reactions.

The same framework would allow to self-assemble modular tissues of logic circuits as discussed in Section IV B, with 'wiring' molecules diffusing only among adjacent compartments. Indeed, Fuchslin et al. [28] have demonstrated that it is viable to construct large-scale (in fact scalable) logic circuits, such as $N$-bit multipliers, by programmed self-assembly of small circuit modules: the study demonstrates that it is possible to transfer part of the algorithm to be performed from the circuitry itself into the arrangement of limited number (something in between four to eight) of different types of logic units - and thus into the self-assembly process of these building blocks. Again, the self-assembly process is driven by the matching of surface markers on the building blocks. The key point is that the building blocks interact only locally. Scalability, the capability to solve arbitrarily large multiplications, results from pattern generation by self-assembly that can lead to arbitrary large structures. The combination of logic with geometry enables inductive generalization: the solution of a potentially infinite class of problems from a finite number of problem instances.

Note that the formal frameworks described in Section III do not offer direct means of expressing compartment association as used in the approaches of Fuchslin et al. [28] and Reller [60]. Martin-Vide et al. [49] have developed tissue P systems, where the connectivity of compartments is given by an adjacency matrix. Using this framework, it would be possible to express the dynamics that occur within an assembled tissue.

\section{DISCUSSION}

In this article, we have demonstrated several theoretical frameworks that capture reactions in and of compartmentalized systems, applied to recent case studies of compartmented systems in synthetic biology. The examples demonstrate how compartmentalization can be used in synthetic biological systems to implement modularization and data hiding similar to encapsulation in traditional programming.

In general, this work advances the availability of biological and chemical programming languages and formal frameworks. In particular, we aim at analyzing the facility with which one can use the various formalisms to program and debug complex nano-bio systems. Table I shows a comparison of the different frameworks in terms of features and applicability. Clearly, all frameworks have different stengths and weaknesses, and there is currently no unifying framework that meets the requirements of all presented case studies.

Importantly, none of the current frameworks capture both compartment association (as found in tissue $\mathrm{P}$ systems) and compartment transformations such as splitting and merging (as found in the brane and chemtainer calculi). For example, tissue $\mathrm{P}$ systems and the artificial vesicle tissue discribed in Sec. II and IV D currently follow the logic of static self-assembly in the sense that a tissue is assembled prior to being used for its functional purpose. In contrast, one could envisage dynamic self-assembly where the self-assembly dynamics of the tissue is coupled with its functionality. Such coupled dynamics would allow one for example to design - at least in theory - the controlled and release and replacement of "emptied" or "burned out" compartments. Such dynamic self-assembly intrinsically enables healing: a mesoscopic (with respect to the size of the self-assembling components) perturbation can heal out. In addition, chemtainers that are emptied during the processing can be continuously refilled by dynamic self-assembly. Last but not least, if the fitness landscape changes during the processing (even as a consequence of the process itself) a dynamically assembled structure may be able to react and adapt to the changed landscape. The difference between programming and running a programmed self-assembled system is blurred. Note that such an adaptation can happen in a rather simple manner, e.g. by simply changing the content of the chemtainers one brings into the system over the course of refilling. However, general formal methods able to express such dynamic self-assembly have yet to be developed.

In some case studies (e.g. the computing bacteria of Sec. IV C), we have formalized synthetic biology systems that had already been designed and even realized experimentally. We believe, however, that the true potential of the presented methods lies in enabling algorithms and software tools for the computer assisted design of complex synthetic biological designs de novo. In fact, Reller [60], Weyland et al. [83] and Fellermann and Cardelli [24] developed algorithms for the automated inference of chemical synthesis pathways in programmable nano-bioreactors.

To illustrate the potential benefits of computational design based on formal frameworks, we give the following example: Lui et al. [46] use a simple and taylored computational model to guide the engineering of synthetic polymer-- 
Framework features

Intra-compartment reactions
Trans-compartment reactions (diffusion)
Inter-compartment reactions (e.g. merge, split)
Hierarchical compartmentalization
Compartment association
Explicit DNA tag operations
Applicability on case studies
Programmable chemical synthesis (Sec. IV A)
Modular molecular computing (Sec. IV B)
Modular bacterial computing (Sec. IV C)
Programmable nanoreactor tissue (Sec. IV D)

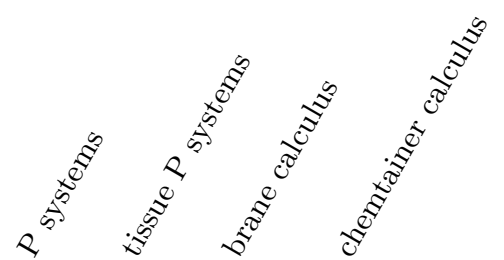

$\begin{array}{llll}\checkmark & \checkmark & \checkmark & \checkmark \\ \checkmark & \checkmark & \checkmark & -(1) \\ \checkmark & - & \checkmark & \checkmark \\ \checkmark & - & \checkmark & \checkmark \\ - & \checkmark & - & - \\ - & - & - & \checkmark \\ - & - & \checkmark & \checkmark \\ - & - & \checkmark & \checkmark \\ \checkmark & \checkmark & \checkmark & -(1) \\ - & \checkmark & - & -\end{array}$

Table I. Feature comparison of the discussed frameworks. (1) Not adding transmembrane diffusion was a design decision in the chemtainer calculus with respect to its original application. Such transitions could be easily added if desired for other applications.

bacteria mictures exhibiting targeted quorum sensing and cell clustering. In their work, polymers were synthesized prior to introduction into the cell milieu. Subsequently, Magennis et al. [47] demonstrate via a laborious trial-anderror approach that polymers can be templated by the cells themselves, which exceeds the scope of the computational model. In order to expand Magennis et al. [47] into a generic and scalable approach, generic tools for rational forward design would be needed - based on our chemtainer calculus or simmilar modelling approaches.

While it may be questionable whether naturally evolved systems can be directly mapped in an efficient and practical way into formal languages, this potential limitation is greatly diminished when considering engineered systems such as those being pursued in Synthetic Biology (both from the bottom up and top down). This is so because there is ample opportunity for "co-designing" the biological/biochemical substrate and the logic circuitry it is meant to implement. Co-design is a technique used in the ICT industry whereby the hardware and the software that will run on it are designed simultaneously thus enabling the optimal tuning of one to each other. This contrasts with the case where generic hardware is designed and built prior to understanding what software will be run on it. As a consequence, software ends-up executed on an already (over)constrained architecture. Thus the importance of advancing our understanding of formal computational methods simultaneously with building new biological and chemical information processing architectures.

Currently, we are working in implementing an integrated development environment for compartmentalized biological systems based on the discussed formalisms, similar to the one Romero-Campero et al. [62] and Blakes et al. [8] have developed for $\mathrm{P}$ systems. The platform intends to facilitate the design, simulation, verification, and ultimately the rapid prototyping of synthetic biological systems [67].

Advances in our understanding of chemical and biological complexity as well as in the availability of powerful computational formalism for the rapid model prototyping of programmable biological and chemical cells are opening a new frontier in computing science: algorithmic living matter. The availability of new theoretical tools as well as practical implementations for algorithmic living matter, we believe, will lead to a new revolution in computing science based on "programmable everywhere", namely, the ability to program all kinds of materials in all kinds of environments at all scales.

\section{ACKNOWLEDGMENTS}

The research leading to these results has received partial funding from the European Community's Seventh Framework Programme (FP7/2007-2013) under grant agreement n²49032 (MATCHIT), and the UK's EPSRC under grants 
EP/J004111/1 (AUDACIOUS), EP/L001489/1 (TAURUS), and EP/I031642/1 (ROADBLOCK).

[1] M. Amos, P. Dittrich, J. McCaskill, and S. Rasmussen. 2011. Biological and Chemical Information Technologies. In Proceedings from the 2nd European Future Technologies Conference and Exhibition 2011 (FET 11). Procedia Computer Science, 56-60.

[2] G. Bacci and M. Miculan. 2012. Measurable stochastics for Brane Calculus. Theor. Comp. 431 (2012), $117-136$.

[3] P. A. Beales and T. K. Vanderlick. 2007. Specific Binding of Different Vesicle Populations by the Hybridization of Membrane-Anchored DNA. J. Phys. Chem. A 111, 49 (Dec. 2007), 12372-12380. DOI:http://dx.doi.org/10.1021/ jp075792z

[4] M. D. Best. 2009. Click Chemistry and Bioorthogonal Reactions: Unprecedented Selectivity in the Labeling of Biological Molecules. Biochemistry 48, 28 (July 2009), 6571-6584. DOI:http://dx.doi.org/10.1021/bi9007726

[5] P. L. Biancaniello, J. C. Crocker, D. A. Hammer, and V. T. Milam. 2007. DNA-Mediated Phase Behavior of Microsphere Suspensions. Langmuir 23, 5 (Feb. 2007), 2688-2693. DOI:http://dx.doi.org/10.1021/la062885j

[6] P. L. Biancaniello, A. J. Kim, and J. C. Crocker. 2005. Colloidal Interactions and Self-Assembly Using DNA Hybridization. Physical Review Letters 94, 5 (Feb. 2005), 058302. DOI:http://dx.doi.org/10.1103/PhysRevLett.94.058302

[7] J. Bibette, F. Leal Calderon, and P. Poulin. 1999. Emulsions: basic principles. Reports on Progress in Physics 62,6 (June 1999), 969. DOI:http://dx.doi.org/10.1088/0034-4885/62/6/203

[8] J. Blakes, J. Twycross, F. J. Romero-Campero, and N. Krasnogor. 2011. The Infobiotics Workbench: an integrated in silico modelling platform for Systems and Synthetic Biology. Bioinformatics 27, 23 (Dec. 2011), 3323-3324. DOI: http://dx.doi.org/10.1093/bioinformatics/btr571

[9] P.-Y. Bolinger, D. Stamou, and H. Vogel. 2004. Integrated Nanoreactor Systems: Triggering the Release and Mixing of Compounds Inside Single Vesicles. Journal of the American Chemical Society 126, 28 (July 2004), 8594-8595. DOI: http://dx.doi.org/10.1021/ja049023u

[10] J. S. Bonifacino and B. S. Glick. 2004. The Mechanisms of Vesicle Budding and Fusion. Cell 116, 2 (Jan. 2004), 153-166. DOI : http://dx.doi.org/10.1016/S0092-8674(03)01079-1

[11] C. Boyer and J. A. Zasadzinski. 2007. Multiple Lipid Compartments Slow Vesicle Contents Release in Lipases and Serum. ACS Nano 1, 3 (Oct. 2007), 176-182. DOI:http://dx.doi.org/10.1021/nn7002025

[12] I. Canton and G. Battaglia. 2012. Endocytosis at the nanoscale. Chemical Society Reviews 41, 7 (2012), 2718. DOI: http://dx.doi.org/10.1039/c2cs15309b

[13] L. Cardelli. 2005. brane calculi - Interactions of Biological Membranes. In Computational Methods in Systems Biology, V. Danos and V. Schachter (Eds.). Springer, 257-278.

[14] L. Cardelli. 2011. Strand algebras for DNA computing. Nat. Comput. 10 (2011), 407-428. DOI:http://dx.doi.org/10. 1007/s11047-010-9236-7

[15] P. Carrara, P. Stano, and P. L. Luisi. 2012. Giant Vesicles Colonies: A Model for Primitive Cell Communities. ChemBioChem 13, 10 (2012), 14971502. DOI:http://dx.doi.org/10.1002/cbic. 201200133

[16] F. Caschera, P. Stano, and P. L. Luisi. 2010. Reactivity and fusion between cationic vesicles and fatty acid anionic vesicles. Journal of Colloid and Interface Science 345, 2 (May 2010), 561-565. DOI:http://dx.doi.org/10.1016/j.jcis.2010. 01.059

[17] F. Caschera, T. Sunami, T. Matsuura, H. Suzuki, and M. Hanczyc. 2011. Programmed Vesicle Fusion Triggers Gene Expression. Langmuir 27, 21 (2011), 13082-13090.

[18] V. Cavalli, M. Corti, and J. Gruenberg. 2001. Endocytosis and signaling cascades: a close encounter. FEBS Letters 498, 23 (June 2001), 190-196. DOI:http://dx.doi.org/10.1016/S0014-5793(01)02484-X

[19] Y. M. Chan, B. van Lengerich, and S. G. Boxer. 2009. Effects of linker sequences on vesicle fusion mediated by lipidanchored DNA oligonucleotides. Proceedings of the National Academy of Sciences 106, 4 (Jan. 2009), 979-984. DOI: http://dx.doi.org/10.1073/pnas.0812356106

[20] J. C. Chaplin, N. A. Russell, and N. Krasnogor. 2012. Implementing conventional logic unconventionally: Photochromic molecular populations as registers and logic gates. Biosystems 109, 1 (July 2012), 35-51. DOI:http://dx.doi.org/10. 1016/j.biosystems.2011.12.005

[21] Y. Chen, N. Dalchau, N. Srinivas, A. Phillips, L. Cardelli, D. Soloveichik, and G. Seelig. 2013. Programmable chemical controllers made from DNA. Nature Nanotechnology 8, 10 (Oct. 2013), 755-762. DOI:http://dx.doi.org/10.1038/ nnano.2013.189

[22] D. T. Chiu, C. F. Wilson, F. Ryttsn, A. Strmberg, C. Farre, A. Karlsson, S. Nordholm, A. Gaggar, B. P. Modi, A. Moscho, R. A. Garza-Lpez, O. Orwar, and R. N. Zare. 1999. Chemical Transformations in Individual Ultrasmall Biomimetic Containers. Science 283, 5409 (March 1999), 1892-1895. DOI:http://dx.doi.org/10.1126/science.283.5409.1892

[23] S. Cobbe, S. Connolly, D. Ryan, L. Nagle, R. Eritja, and D. Fitzmaurice. 2003. DNA-Controlled Assembly of ProteinModified Gold Nanocrystals. The Journal of Physical Chemistry B 107, 2 (Jan. 2003), 470-477. DOI:http://dx.doi. org/10.1021/jp021503p

[24] H. Fellermann and L. Cardelli. 2014. Programmable chemistry in DNA addressable bioreactors. R. Soc. Interface (2014).

[25] S. F. Fenz and K. Sengupta. 2012. Giant vesicles as cell models. Integrative Biology 4, 9 (2012), 982. DOI:http: //dx.doi.org/10.1039/c2ib00188h 
[26] C. M. Franzin and P. M. Macdonald. 1997. Detection and Quantification of Asymmetric Lipid Vesicle Fusion Using Deuterium NMR. Biochemistry 36, 9 (March 1997), 2360-2370. DOI:http://dx.doi.org/10.1021/bi9621270

[27] R. M. Fuchslin, A. Dzyakanchuk, D. Flumini, H. Hauser, K. J. Hunt, R. H. Luchsinger, B. Reller, S. Scheidegger, and R. Walker. 2013. Morphological computation and morphological control: Steps toward a formal theory and applications. Artif. Life 19, 1 (Jan. 2013), 9-34. DOI:http://dx.doi.org/10.1162/ARTL_a_00079

[28] R. M. Fuchslin, T. Maeke, U. Tangen, and J. S. McCaskill. 2006. Evolving inductive generalization via genetic self-assembly. Advances in Complex Systems 09, 01n02 (March 2006), 1-29. DOI:http://dx.doi.org/10.1142/S0219525906000598

[29] Z. J. Gartner and C. R. Bertozzi. 2009. Programmed assembly of 3-dimensional microtissues with defined cellular connectivity. Proceedings of the National Academy of Sciences 106, 12 (March 2009), 4606-4610. DOI:http: //dx.doi.org/10.1073/pnas.0900717106

[30] M. Hadorn, E. Boenzli, K. T. Srensen, D. De Lucrezia, M. M. Hanczyc, and T. Yomo. 2013. Defined DNA-Mediated Assemblies of Gene-Expressing Giant Unilamellar Vesicles. Langmuir 29, 49 (Dec. 2013), 15309-15319. DOI:http://dx. doi.org/10.1021/la402621r

[31] M. Hadorn, E. Bonzli, H. Fellermann, P. Eggenberger Hotz, and M. Hanczyc. 2012. Specific and reversible DNA-directed self-assembly of emulsion droplets. Proc. Nat. Acad. Sci. USA 109, 47 (2012).

[32] M. Hadorn and P. Eggenberger Hotz. 2010. DNA-mediated self-assembly of artificial vesicles. PLoS One 5, 3 (2010), e9886.

[33] C. K. Haluska, K. A. Riske, V. Marchi-Artzner, J.-M. Lehn, R. Lipowsky, and R. Dimova. 2006. Time scales of membrane fusion revealed by direct imaging of vesicle fusion with high temporal resolution. Proceedings of the National Academy of Sciences 103, 43 (Oct. 2006), 15841-15846. DOI:http://dx.doi.org/10.1073/pnas.0602766103

[34] K. Ishikawa, K. Sato, Y. Shima, I. Urabe, and T. Yomo. 2004. Expression of a cascading genetic network within liposomes. FEBS Lett. 576 (2004), 387-390.

[35] A. Jesorka and O. Orwar. 2008. Liposomes: Technologies and Analytical Applications. Annual Review of Analytical Chemistry 1, 1 (2008), 801-832. DOI:http://dx.doi.org/10.1146/annurev.anchem.1.031207.112747

[36] S. Kim, M. S. Turker, E. Y. Chi, S. Sela, and G. M. Martin. 1983. Preparation of multivesicular liposomes. Biochimica et Biophysica Acta (BBA) - Biomembranes 728, 3 (March 1983), 339-348. DOI : http://dx.doi.org/10.1016/0005-2736(83) 90504-7

[37] K. M. Koeller and C. Wong. 2000. Complex carbohydrate synthesis tools for glycobiologists: enzyme-based approach and programmable one-pot strategies. Glycobiology 10, 11 (2000), 1157-1169.

[38] H. C. Kolb, M. G. Finn, and K. B. Sharpless. 2001. Click Chemistry: Diverse Chemical Function from a Few Good Reactions. Angewandte Chemie International Edition 40, 11 (2001), 20042021. DOI:http://dx.doi.org/10.1002/ 1521-3773(20010601) 40:11<2004: :AID-ANIE2004>3.0.C0;2-5

[39] H. C Kolb and K. B. Sharpless. 2003. The growing impact of click chemistry on drug discovery. Drug Discovery Today 8, 24 (Dec. 2003), 1128-1137. DOI:http://dx.doi.org/10.1016/S1359-6446(03)02933-7

[40] S. B. Kulkarni, G. V. Betageri, and M. Singh. 1995. Factors affecting microencapsulation of drugs in liposomes. Journal of Microencapsulation 12, 3 (Jan. 1995), 229-246. DOI:http://dx.doi.org/10.3109/02652049509010292

[41] T. Kurpiers and H. D. Mootz. 2009. Bioorthogonal Ligation in the Spotlight. Angewandte Chemie International Edition 48, 10 (2009), 17291731. DOI:http://dx.doi.org/10.1002/anie. 200805454

[42] M. R. Lakin, S. Youssef, L. Cardelli, and A. Phillips. 2012. Abstractions for DNA circuit design. J R Soc Interface 9, 68 (2012), 470-86. DOI:http://dx.doi.org/10.1098/rsif.2011.0343

[43] D. D. Lasic. 1988. The mechanism of vesicle formation. Biochemical Journal 256, 1 (Nov. 1988), 1-11. http://www.ncbi. nlm.nih.gov/pmc/articles/PMC1135360/

[44] M. E. Leunissen, R. Dreyfus, F. C. Cheong, D.d G. Grier, R. Sha, N. C. Seeman, and P. M. Chaikin. 2009. Switchable self-protected attractions in DNA-functionalized colloids. Nature Materials 8, 7 (July 2009), 590-595. D0I:http://dx. doi.org/10.1038/nmat 2471

[45] N. A. Licata and A. V. Tkachenko. 2006. Statistical mechanics of DNA-mediated colloidal aggregation. Physical Review E 74, 4 (Oct. 2006), 041408. DOI :http://dx.doi.org/10.1103/PhysRevE.74.041408

[46] Leong T. Lui, Xuan Xue, Cheng Sui, Alan Brown, David I. Pritchard, Nigel Halliday, Klaus Winzer, Steven M. Howdle, Francisco Fernandez-Trillo, Natalio Krasnogor, and Cameron Alexander. 2013. Bacteria clustering by polymers induces the expression of quorum-sensing-controlled phenotypes. Nature Chemistry 5, 12 (Dec. 2013), 1058-1065. DOI:http: //dx.doi.org/10.1038/nchem.1793

[47] E. Peter Magennis, Francisco Fernandez-Trillo, Cheng Sui, Sebastian G. Spain, David J. Bradshaw, David Churchley, Giuseppe Mantovani, Klaus Winzer, and Cameron Alexander. 2014. Bacteria-instructed synthesis of polymers for selfselective microbial binding and labelling. Nature Materials 13, 7 (July 2014), 748-755. DOI:http://dx.doi.org/10.1038/ nmat3949

[48] J. P. Magnusson, F. Fernndez-Trillo, G. Sicilia, S. G. Spain, and C. Alexander. 2014. Programmed assembly of polymerDNA conjugate nanoparticles with optical readout and sequence-specific activation of biorecognition. Nanoscale 6, 4 (2014), 2368. DOI:http://dx.doi.org/10.1039/c3nr04952c

[49] C. Martin-Vide, G. Paun, J. Pazos, and A. Rodriguez-Paton. 2003. Tissue P systems. Theoretical Computer Science 296, 2 (March 2003), 295-326. DOI : http://dx.doi.org/10.1016/S0304-3975(02) 00659-X

[50] T. Maruyama, H. Yamamura, M. Hiraki, Y. Kemori, H. Takata, and M. Goto. 2008. Directed aggregation and fusion of lipid vesicles induced by DNA-surfactants. Colloids and Surfaces B: Biointerfaces 66, 1 (Oct. 2008), 119-124. D0I: http://dx.doi.org/10.1016/j.colsurfb.2008.05.017

[51] C. A. Mirkin, R. L. Letsinger, R. C. Mucic, and J. J. Storhoff. 1996. A DNA-based method for rationally assembling 
nanoparticles into macroscopic materials. Nature 382, 6592 (Aug. 1996), 607-609. D0I:http://dx.doi.org/10.1038/ $382607 \mathrm{a} 0$

[52] O. Mondragn-Palomino, T. Danino, J. Selimkhanov, L. Tsimring, and J. Hasty. 2011. Entrainment of a Population of Synthetic Genetic Oscillators. Science 333, 6047 (Sept. 2011), 1315-1319. DOI:http://dx.doi.org/10.1126/science. 1205369

[53] P.-A. Monnard. 2003. Liposome-entrapped polymerases as models for microscale/nanoscale bioreactors. J. Membr. Biol. 191, 2 (2003), 87-97. DOI:http://dx.doi.org/10.1007/s00232-002-1046-0

[54] C. Nardin, J. Widmer, M. Winterhalter, and W. Meier. 2001. Amphiphilic block copolymer nanocontainers as bioreactors. Euro. Phys. J. E 4 (2001), 403-410.

[55] V. Noireaux and A. Libchaber. 2004. A vesicle bioreactor as a step toward an artificial cell assembly. Proc. Nat. Acad. Sci. USA 101, 51 (2004), 17669-17674.

[56] S. M. Nomura, K. Tsumoto, T. Hamada, K. Akiyoshi, Y. Nakatani, and K. Yoshikawa. 2003. Gene Expression within Cell-Sized Lipid Vesicles. ChemBioChem 4, 11 (2003), 11721175. DOI:http://dx.doi.org/10.1002/cbic.200300630

[57] G. Paun. 2000. Computing with Membranes. J. Comput. System Sci. 61, 1 (Aug. 2000), 108-143. D0I:http://dx.doi. org/10.1006/jcss.1999.1693

[58] S. Rasmussen, M. Bedau, L. Chen, D. Deamer, D. Krakauer, N. Packard, and P. Stadler (Eds.). 2008. Protocells: Bridging Nonliving and Living Matter. MIT Press, Cambridge, USA.

[59] S. Regot, J. Macia, N. Conde, K. Furukawa, J. Kjelln, T. Peeters, S. Hohmann, E. de Nadal, F. Posas, and R. Sol. 2011. Distributed biological computation with multicellular engineered networks. Nature 469, 7329 (Jan. 2011), 207-211. D0I: http://dx.doi.org/10.1038/nature09679

[60] B. Reller. 2010. Programmable self-assembling spatially heterogeneous micro-reactors. MSc thesis. University of Zurich, Zurich.

[61] A. Richard, V. Marchi-Artzner, M.-N. Lalloz, M.-J. Brienne, F. Artzner, T. Gulik-Krzywicki, M.-A. Guedeau-Boudeville, and J.-M. Lehn. 2004. Fusogenic supramolecular vesicle systems induced by metal ion binding to amphiphilic ligands. Proc. Nat. Acad. Sci. USA 101, 43 (Oct. 2004), 15279-15284. DOI:http://dx.doi.org/10.1073/pnas.0406625101

[62] F. J. Romero-Campero, J. Twycross, M. Cmara, M. Bennett, M. Gheorghe, and N. Krasnogor. 2009. Modular Assembly of cell systems biology models using P systems. International Journal of Foundations of Computer Science 20 , 03 (June 2009), 427-442. DOI:http://dx.doi.org/10.1142/S0129054109006668

[63] R. Roodbeen and J. C. M. van Hest. 2009. Synthetic cells and organelles: compartmentalization strategies. BioEssays 31, 12 (2009), 1299-1308. DOI:http://dx.doi.org/10.1002/bies.200900106

[64] P. W. K. Rothemund. 2006. Folding DNA to create nanoscale shapes and patterns. Nature 440, 7082 (March 2006), 297-302. DOI:http://dx.doi.org/10.1038/nature04586

[65] J. E. Rothman. 1981. The golgi apparatus: two organelles in tandem. Science 213, 4513 (Sept. 1981), 1212-1219. DOI: http://dx.doi.org/10.1126/science.7268428

[66] J. E. Rothman. 1994. Mechanisms of intracellular protein transport. Nature 372, 6501 (Nov. 1994), 55-63. DOI:http: //dx.doi.org/10.1038/372055a0

[67] D. Sanassy, H. Fellermann, N. Krasnogor, S. Konur, L. M. Mierla, M. Gheorghe, C. Ladroue, and S. Kalvala. 2014. Modelling and stochastic simulation of synthetic biological Boolean gates. In Proceedings of the 16nth IEEE International Conference on High Performance Computing and Communications. IEEE Publishing, Paris.

[68] N. C. Seeman. 2003. DNA in a material world. Nature 421, 6921 (Jan. 2003), 427-431. DOI:http://dx.doi.org/10. 1038/nature01406

[69] R. Silva-Rocha and V. de Lorenzo. 2008. Mining logic gates in prokaryotic transcriptional regulation networks. FEBS Letters 582, 8 (April 2008), 1237-1244. DOI:http://dx.doi.org/10.1016/j.febslet.2008.01.060

[70] J. Smaldon, F. J. Romero-Campero, F. F. Trillo, M. Gheorghe, C. Alexander, and N. Krasnogor. 2010. A computational study of liposome logic: towards cellular computing from the bottom up. Systems and Synthetic Biology 4, 3 (Sept. 2010), 157-179. DOI:http://dx.doi.org/10.1007/s11693-010-9060-5

[71] G. Stengel, R. Zahn, and F. Hk. 2007. DNA-Induced Programmable Fusion of Phospholipid Vesicles. Journal of the American Chemical Society 129, 31 (Aug. 2007), 9584-9585. D0I:http://dx.doi.org/10.1021/ja073200k

[72] B. Stdler, D. Falconnet, I. Pfeiffer, F. Hk, and J. Vrs. 2004. Micropatterning of DNA-Tagged Vesicles. Langmuir 20, 26 (Dec. 2004), 11348-11354. DOI:http://dx.doi.org/10.1021/la0482305

[73] T. Sunami, F. Caschera, Y. Morita, T. Toyota, K. Nishimura, T. Matsuura, H. Suzuki, M. M. Hanczyc, and T. Yomo. 2010. Detection of Association and Fusion of Giant Vesicles Using a Fluorescence-Activated Cell Sorter. Langmuir 26, 19 (Oct. 2010), 15098-15103. DOI:http://dx.doi.org/10.1021/la102689v

[74] A. Tamsir, J. J. Tabor, and C. A. Voigt. 2011. Robust multicellular computing using genetically encoded NOR gates and chemical /'wires/'. Nature 469, 7329 (Jan. 2011), 212-215. DOI : http://dx.doi.org/10.1038/nature09565

[75] H. Terasawa, K. Nishimura, H. Suzuki, T. Matsuura, and T. Yomo. 2012. Coupling of the fusion and budding of giant phospholipid vesicles containing macromolecules. Proc. Nat. Acad. Sci. USA 109, 16 (April 2012), 5942-5947. DOI: http://dx.doi.org/10.1073/pnas. 1120327109

[76] C. K. Tison and V. T. Milam. 2007. Reversing DNA-Mediated Adhesion at a Fixed Temperature. Langmuir 23, 19 (Sept. 2007), 9728-9736. DOI:http://dx.doi.org/10.1021/la700601j

[77] V. P. Torchilin. 2005. Recent advances with liposomes as pharmaceutical carriers. Nature Reviews Drug Discovery 4,2 (Feb. 2005), 145-160. DOI:http://dx.doi.org/10.1038/nrd1632

[78] M.-P. Valignat, O. Theodoly, J. C. Crocker, W. B. Russel, and P. M. Chaikin. 2005. Reversible self-assembly and directed assembly of DNA-linked micrometer-sized colloids. Proceedings of the National Academy of Sciences of the United States 
of America 102, 12 (March 2005), 4225-4229. DOI:http://dx.doi.org/10.1073/pnas.0500507102

[79] A. Varki. 1993. Biological roles of oligosaccharides: all of the theories are correct. Glycobiology 3, 2 (April 1993), 97-130. DOI:http://dx.doi.org/10.1093/glycob/3.2.97

[80] G. Villar, A. D. Graham, and H. Bayley. 2013. A Tissue-Like Printed Material. Science 340, 6128 (April 2013 ), 48-52. DOI:http://dx.doi.org/10.1126/science.1229495

[81] P. Waage and C. M. Gulberg. 1986. Studies concerning affinity. Journal of Chemical Education 63, 12 (Dec. 1986$), 1044$. DOI:http://dx.doi.org/10.1021/ed063p1044

[82] S. A. Walker, M. T. Kennedy, and J. A. Zasadzinski. 1997. Encapsulation of bilayer vesicles by self-assembly. Nature 387, 6628 (May 1997), 61-64. DOI :http://dx.doi.org/10.1038/387061a0

[83] M. S. Weyland, H. Fellermann, M. Hadorn, D. Sorek, D. Lancet, S. Rasmussen, and R. M. Fuchslin. 2013. The MATCHIT Automaton: Exploiting Compartmentalization for the Synthesis of Branched Polymers. Computational and Mathematical Methods in Medicine 2013 (Dec. 2013), 467428. DOI :http://dx.doi.org/10.1155/2013/467428

[84] G. M. Whitesides and M. Boncheva. 2002. Beyond molecules: Self-assembly of mesoscopic and macroscopic components. Proc. Nat. Acad. Sci. USA 99, 8 (April 2002), 4769-4774. DOI:http://dx.doi.org/10.1073/pnas.082065899

[85] E. Winfree, F. Liu, L. A. Wenzler, and N. C. Seeman. 1998. Design and self-assembly of two-dimensional DNA crystals. Nature 394, 6693 (Aug. 1998), 539-544. DOI :http://dx.doi.org/10.1038/28998

[86] H. Zhang, G. Wang, and H. Yang. 2011. Drug delivery systems for differential release in combination therapy. Expert Opinion on Drug Delivery 8, 2 (Feb. 2011), 171-190. DOI:http://dx.doi.org/10.1517/17425247.2011.547470

XXX 2014XXX 2014 